\title{
A comprehensive flow-cytometry-based immunophenotypic characterization of Burkitt-like lymphoma with 11q aberration
}

Grzegorz Rymkiewicz ${ }^{1,2}$, Beata Grygalewicz ${ }^{3}$, Magdalena Chechlinska ${ }^{4}$, Katarzyna Blachnio ${ }^{1}$, Zbigniew Bystydzienski ${ }^{1}$, Joanna Romejko-Jarosinska ${ }^{5}$, Renata Woroniecka ${ }^{3}$, Michalina Zajdel ${ }^{4}$, Katarzyna Domanska-Czyz ${ }^{5}$, David Martin-Garcia ${ }^{6}$, Ferran Nadeu ${ }^{6}$, Pawel Swoboda ${ }^{4}$, Jolanta Rygier ${ }^{3}$, Barbara Pienkowska-Grela ${ }^{3}$, Jan Konrad Siwicki ${ }^{4}$, Monika Prochorec-Sobieszek ${ }^{2}$, Itziar Salaverria ${ }^{6,8}$, Reiner Siebert ${ }^{7,8}$ and Jan Walewski ${ }^{5,8}$

${ }^{1}$ Flow Cytometry Laboratory, Department of Pathology and Laboratory Diagnostics, Maria Sklodowska-Curie Institute-Oncology Center, Warsaw, Poland; ${ }^{2}$ Pathology Laboratory, Department of Pathology and Laboratory Diagnostics, Maria Sklodowska-Curie Institute-Oncology Center, Warsaw, Poland; ${ }^{3}$ Cytogenetics Laboratory, Department of Pathology and Laboratory Diagnostics, Maria Sklodowska-Curie Institute-Oncology Center, Warsaw, Poland; ${ }^{4}$ Department of Immunology, Maria Sklodowska-Curie Institute-Oncology Center, Warsaw, Poland; ${ }^{5}$ Department of Lymphoid Malignancies, Maria Sklodowska-Curie Institute-Oncology Center,

Warsaw, Poland; ${ }^{6}$ Hematopathology Unit, Hospital Clínic, Institut d'Investigacions Biomèdiques August Pi i Sunyer (IDIBAPS), CIBERONC, University of Barcelona, Barcelona, Spain and ${ }^{7}$ Institute of Human Genetics, University Ulm and Ulm University Medical Center, Ulm, Germany

\begin{abstract}
We previously described a subset of $M Y C$ translocation-negative aggressive B-cell lymphomas resembling Burkitt lymphoma, characterized by proximal gains and distal losses in chromosome 11. In the 2016 WHO classification, these MYC-negative lymphomas were recognized as a new provisional entity, 'Burkitt-like lymphoma with 11q aberration'. Here we present an immunophenotype analysis of Burkitt-like lymphomas with $11 q$ aberration. Cells were acquired by fine needle aspiration biopsy from 10 young adult patients, $80 \%$ of whom presented recurrence-free 5-year survival. Twenty-three $M Y C$-positive Burkitt lymphomas, including three carrying both $M Y C$ rearrangement and 11q aberration, served as controls. By immunohistochemistry, all Burkittlike lymphomas with 11q aberration were CD20+/CD10+/BCL6+/BCL2 - /MUM1 - /MYC+/EBV -, usually LMO2 +/CD44 - /CD43 - and sometimes CD56+, and showed high proliferation rate. By flow cytometry, Burkitt-like lymphoma with 11q aberration immunophenotypically resembled $M Y C$-positive Burkitt lymphoma, except for significantly (adjusted $\boldsymbol{P}<0.001)$ more frequent CD $38^{\text {higher }}$ expression in Burkitt lymphoma $(91 \% M Y C$-positive Burkitt lymphoma vs 10\% Burkitt-like lymphoma with 11q aberration), more frequently diminished CD45 expression in Burkitt lymphoma (74\% vs 10\%), an exclusive CD16/CD56 and highly restricted CD8 expression in Burkitt-like lymphoma with $11 q$ aberration $(60 \%$ vs $0 \%$ and $40 \%$ vs $4 \%$, respectively). We showed high diagnostic accuracy and effectiveness of flow cytometry in Burkitt lymphoma. CD16/CD56 expression without CD38 higher and the lack of CD16/CD56 with CD38 ${ }^{\text {higher }}$ expression proves to be a reliable, fast, and cost-effective method for diagnosing 11q aberration and $M Y C$ rearrangements in CD10(+) aggressive lymphomas, respectively. In addition, we confirmed a pattern of an inverted duplication with telomeric loss of 11q, as a recurrent 11q abnormality, but one case presented alternative changes, possibly resulting in an equivalent molecular effect. Our findings reveal similarities along with subtle but essential differences in the immunophenotype of Burkitt-like lymphoma with 11q aberration and MYC-positive Burkitt lymphoma, important for the differential diagnosis, but also for understanding the pathogenesis of Burkitt-like lymphoma with 11q aberration.

Modern Pathology (2018) 31, 732-743; doi:10.1038/modpathol.2017.186; published online 12 January 2018
\end{abstract}

Correspondence: Dr G Rymkiewicz, MD, PhD, Flow Cytometry Laboratory, Department of Pathology and Laboratory Diagnostics, Maria Sklodowska-Curie Institute-Oncology Center, 5 Roentgen Street, Warsaw 02-781, Poland.

E-mail: grzegorzrymkiewicz@coi.waw.pl

${ }^{8}$ These authors contributed equally to this work.

Received 20 July 2017; revised 20 October 2017; accepted 20 October 2017; published online 12 January 2018 
We have previously described ${ }^{1,2}$ a new entity similar to Burkitt lymphoma with recurrent chromosome 11q aberrations and no detectable $M Y C$ translocation $\left(M Y C^{-}\right)$. By metaphase analyses, these $11 \mathrm{q}$ aberrations mostly present as $\operatorname{dup}(11)(q 23 q 13),{ }^{1}$ and by interphase FISH and high-resolution copy-number arrays, as proximal gains and telomeric losses in $11 \mathrm{q}^{2}$ A similar pattern of $11 \mathrm{q}$ aberrations was observed in $M Y C$-negative post-transplant Burkitt lymphoma. ${ }^{3}$ Remarkably, gene expression analyses of mRNA and miRNA showed major similarities in transcriptional profiles to $M Y C$-positive Burkitt lymphoma. ${ }^{2,4}$ Consequently, in the updated 2016 WHO classification, these lymphomas were recognized as a provisional entity $M Y C$-negative 'Burkittlike lymphoma with $11 \mathrm{q}$ aberration'. ${ }^{5}$

MYC-positive Burkitt lymphoma has typical histopathological and immunohistochemical features (CD20+/CD10+/BCL6+/BCL2 - /MUM1 - /MYC+/CD44 -/CD43+/Ki-67>95\%) in most cases, enabling differentiation from other, more common aggressive B-cell lymphomas. ${ }^{6-8}$ Recently, lack of LMO2 expression by immunohistochemistry was found to be significantly associated with $M Y C$ translocations in $\mathrm{CD} 10(+)$ aggressive B-cell lymphomas, including $M Y C$-positive Burkitt lymphoma, ${ }^{9}$ which is consistent with gene expression profiling studies, that have shown low levels of $\mathrm{LMO} 2$ gene expression in $M Y C$-positive Burkitt lymphoma. ${ }^{8}$ Rare Burkitt-like lymphoma with $11 \mathrm{q}$ aberration have recently been characterized with the use of immunohistochemistry, ${ }^{1-4}$ but a detailed flow cytometry analysis of these cases compared to $M Y C$-positive Burkitt lymphoma is missing.

In the current study, we present patients with highly aggressive Burkitt-like lymphoma with 11q aberration, characterized by a spectrum of histological features consistent with $M Y C$-positive Burkitt lymphoma and a recurrent $11 \mathrm{q}$ aberrations, diagnosed before the 2016 update of WHO classification, mostly as $M Y C$-negative Burkitt lymphoma and treated according to $M Y C$-positive Burkitt lymphoma regimens, at a single institution. We show here that Burkitt-like lymphoma with $11 \mathrm{q}$ aberrations is characterized by a distinct flow cytometry and immunohistochemical immunophenotype.

\section{Materials and methods}

\section{Patients}

Among 82 consecutive adult Burkitt lymphoma patients diagnosed at Maria Sklodowska-Curie Institute-Oncology Center between 2000 and 2014 by the standard histopathology and immunohistochemistry, flow cytometry, conventional cytogenetics, and FISH; 10 cases $(12 \%), 9$ male/ 1 female, median age 27 (18-62) years, HIV/EBV-negative, without bone marrow and cerebrospinal fluid involvement, had no BCL2/BCL6/MYC rearrangements, but displayed a

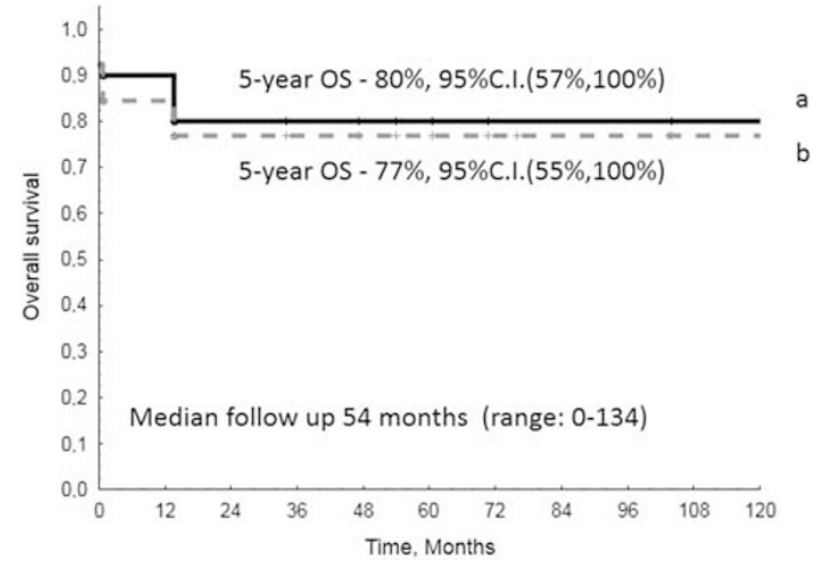

Figure 1 Overall survival from diagnosis, estimated by the Kaplan-Meier method. (a) (continuous line) $M Y C^{-}$Burkitt-like lymphoma with 11q aberration patients, $(n=10)$; (b) (dotted line) All patients with chromosome $11 \mathrm{q}$ aberrations: $M Y C^{-}$and $M Y C^{+}$ lymphomas $(n=13)$.

typical $11 \mathrm{q}$ aberration pattern. The diagnostic material for cytological smears, flow cytometry, conventional cytogenetics, FISH, and copy-number arrays was obtained by fine needle aspiration biopsy as described in Supplementary Methods. Patients were treated with either modified R-CODOX-M/R-IVAC regimen ${ }^{10}$ (Rituximab, fractionated cyclophosphamide, vincristine, doxorubicin, and high-dose methotrexate alternating with fractionated ifosfamide, etoposide and high-dose cytarabine, along with intrathecal methotrexate and cytarabine) or GMALL-B-ALL/NHL2002 protocol ${ }^{11}$ (Rituximab, fractionated cyclophosphamide (or ifosfamide), vincristine, methotrexate, cytarabine, teniposide, and prednisone or doxorubicin). At the last follow-up, 8 out of 10 Burkitt-like lymphoma with 11q aberration patients were alive (Figure 1). Median follow-up was 54 months (range 0-134), and 5-year Overall Survival was $80 \%$ (95\% confidence interval $(55 \%, 100 \%)$ ). Patient and tumor characteristics are shown in Table 1. Twenty-three $M Y C$-positive Burkitt lymphoma patients with simple karyotypes and $M Y C$ rearrangement detected by FISH (19 male/4 female, median age 35 (18-63) years, HIV-negative), including three also carrying $11 \mathrm{q}$ aberration served as immunophenotype controls. Two out of the three patients with Burkitt lymphoma carrying both $M Y C$ rearrangement and $11 \mathrm{q}$ aberration are alive (Figure 1).

\section{Morphological and Immunohistochemical Characterization}

Based on histopathology, all 10 patients were morphologically diagnosed with Burkitt lymphoma. To evaluate cytomorphology of lymphoma cells, cytological smears from fine needle aspiration biopsy were used similarly as in histopathological examination. ${ }^{4}$ Immunohistochemistry and FISH for the Epstein-Barr virus-encoded small RNA (EBER) were performed on 
Table 1 Patient and tumor characteristics

\begin{tabular}{|c|c|c|c|c|c|c|c|c|c|c|c|}
\hline No./A/G & $P S$ & $C S$ & $B$ & $B M / C N S$ & Bulk & Site of involvement & $L D H>U N V$ & $I P I$ & Treatment & Response & Status/Last FU (mo) \\
\hline $1 / 18 / \mathrm{M}^{\sim}$ & 1 & I & No & No & No & $\mathrm{LNc}^{1}$ & No & 1 & GMALL-B-ALL/NHL2002 & CR & ANED (104) \\
\hline $2 / 25 / \mathrm{M}^{\sim}$ & 2 & IV & Yes & No & $\mathrm{Yes}^{\wedge}$ & $\mathrm{LN} / \mathrm{ab}^{1}$ & Yes & 3 & CODOX-M/IVAC & PRpd & DOD (14) \\
\hline $3 / 37 / \mathrm{F}^{\sim}$ & 1 & I & No & No & Yes & $\mathrm{LNc}^{1}$ & No & 0 & GMALL-B-ALL/NHL2002 & CR & ANED (131) \\
\hline $4 / 23 / \mathrm{M}$ & 0 & I & No & No & No & $\mathrm{T}^{1}$ & No & 0 & GMALL-B-ALL/NHL2002 & $\mathrm{CR}$ & ANED (54) \\
\hline $5 / 22 / \mathrm{M}^{\sim}$ & 1 & I & No & No & Yes & LNinq $^{1}$ & Yes & 1 & R-CHOP, RT; CODOX-M/IVAC & PRpd; CR & ANED (134) \\
\hline $6 / 32 / \mathrm{M}$ & 1 & I & No & No & No & $\mathrm{T}^{1}$ & No & 0 & GMALL-B-ALL/NHL2002 & $\mathrm{CR}$ & ANED (34) \\
\hline 8/29/M & 0 & I & No & No & No & $\mathrm{LNc}^{2}$ & No & 0 & R-CODOX-M/R-IVAC, ESHAP & CR & ANED (71) \\
\hline $9 / 62 / \mathrm{M}$ & 1 & IV & No & No & Yes $^{\wedge}$ & $\mathrm{LN} / \mathrm{ab}^{2}$ & Yes & 3 & GMALL-B-ALL/NHL2002 & CR & ANED (47) \\
\hline $10 / 40 / \mathrm{M}$ & 1 & IV & Yes & No & $\mathrm{Yes}^{\wedge}$ & LN/ab ${ }^{1}$ & Yes & 2 & GMALL-B-ALL/NHL2002 & CR & ANED (60) \\
\hline $11 / 20 / \mathrm{M}$ & 4 & IV & No & No & Yes $^{\wedge}$ & $\mathrm{LN} / \mathrm{ab}^{1}$ & Yes & 4 & GMALL-B-ALL/NHL2002 & CR & TRM, Autopsy (1) \\
\hline $12 / 20 / \mathrm{M}$ & 1 & I & No & No & No & $\mathrm{LNc}^{1}$ & Yes & 1 & GMALL-B-ALL/NHL2002 & CR & ANED (103) \\
\hline $13 / 48 / \mathrm{F}$ & 2 & IV & No & No & Yes & $\mathrm{LN} / \mathrm{ab}^{2}$ & Yes & 4 & GMALL-B-ALL/NHL2002 & PR & TRM, Autopsy (1) \\
\hline $14 / 25 / \mathrm{M}$ & 0 & I & No & No & No & $\mathrm{T}^{1}$ & Yes & 1 & GMALL-B-ALL/NHL2002 & CR & ANED (76) \\
\hline
\end{tabular}

No., case number; A/G, Age (years)/Gender-M, male; F, female; Patients No. 1, 2, 3, and 5 were previously reported cases, and correspond to cases 1 , 2 , 3 , and 4 in Pienkowska-Grela et al, ${ }^{1}$ and cases 4, 5, 6 and 7 in Salaverria et al, ${ }^{2}$ respectively; *Patient No. 7 was withdrawn due to unusual 11q aberration; Patients were HIV/HCV/HBV negative; PS, Performance Status; CS, Ann Arbor Stage of disease; B, B symptoms; BM, Bone Marrow involvement; CNS, Central Nervous System involvement; Bulk, tumor $>7 \mathrm{~cm}$ in the greatest dimension, $\wedge$ tumor $>20 \mathrm{~cm}$ in the greatest dimension; LN, lymph node: c, cervical; ing, inguinal; ab, abdominal presentation of disease; T, tonsil; ${ }^{1}$ one enlarged lymph node/tumor, ${ }^{2}$ a few neighboring, enlarged lymph nodes/tumors; LDH $>$ UNV, lactate dehydrogenase elevated above the upper normal value; IPI, International Prognostic Index score; GMALL-B-ALL/NHL2002 regimen (GMALL-German Multicenter Adult ALL Study Group), the alternate use of drugs (Rituximab, fractionated cyclophosphamide (or ifosfamide), vincristine, methotrexate, cytarabine, teniposide, and prednisone or doxorubicin (CNS prophylaxis consisted of triple intrathecal methotrexate, cytarabine, and dexamethasone); CODOX-M/IVAC regimen, fractionated cyclophosphamide, vincristine, doxorubicin, and high-dose methotrexate alternating with fractionated ifosfamide, etoposide, and high-dose cytarabine, along with intrathecal methotrexate and cytarabine; R, Rituximab; RT, Radiation therapy; ESHAP, Etoposide, methylprednisone, cytarabine, cysplatin; R-CHOP, Rituximab, cyclophosphamide, doxorubicin, vincristine, and prednisone; IVAC, fractionated ifosfamide, etoposide, and high-dose cytarabine; PD, Progressive Disease; CR, Complete Remission; PRpd, Partial Remission followed by PD; PR, Partial Remission; ANED, Alive, no evidence of disease; DOD, Died of Disease progression; TRM, Treatment Related Mortality; FU, follow-up (months after the final diagnosis or to death); Horizontal line separates cases into: MYC(-) (above) and MYC(+) lymphomas (below). CS and IPI score were evaluated by standard criteria. 
formalin-fixed, paraffin-embedded tissues, as described by Zajdel et al. ${ }^{4}$ and in Supplementary Methods. Immunohistochemistry was performed using the EnVision Detection Systems FLEX kit (Dako, Carpinteria, CA, USA, code K 8000) or ultraView Universal DAB Detection kit (Ventana Medical Systems, Tucson, USA, catalog no. 760-500) and, if necessary, antigen-retrieval technique was applied for each monoclonal antibody specific for: CD20, CD10, BCL6, BCL2, LMO2, MYC, MUM1, CD43, CD44, CD56 (two different clones of monoclonal antibodies, mouse and rabbit), Ki-67, cyclin D1, CD3, CD5, and TdT (Supplementary Table 1).

\section{Flow Cytometry Immunophenotyping, Cytology, and Cell Staining}

Immunophenotype was also determined by flow cytometry of cellular suspension obtained by fine needle aspiration biopsy or by ultrasound-guided fine needle aspiration biopsy (cases with bulky abdominal mass, stomach, and abdominal lymph node involvement) of the involved lymph nodes, tonsils, and extranodal tumors performed by a hematopathologist. Cells from patients with histopathologically confirmed or suspected of Burkitt lymphoma, were incubated with a panel of monoclonal antibodies ${ }^{1,4,12}$ (for staining procedure and a list of antibodies see Supplementary Methods and Supplementary Table 2). Antigen expression (Figures 2 and 3, and Supplementary Table 4) was quantified by FACSCalibur and FACSCanto II cytometers (Becton Dickinson, BD), and was categorized according to the percentages of positive cells into three groups, marked: '(-)' - no expression ( $<20 \%$ of neoplastic cells), '(+/-)' - expression in $>20 \%$ but $<100 \%$ of cells, and ' $(+)$ ' - in $100 \%$ of lymphoma cells. A quantitative expression of CD (19/20/22/23/52/79 $/ / 81)$, FMC7, HLA-DR, BCL6, and $\operatorname{CD}(5 / 25 / 38 / 43 / 44 / 45 / 52 / 62 \mathrm{~L} / 71 / 200)$, BCL2, and $\mathrm{CD}(16 / 56$ and 56) in neoplastic cells was evaluated as median fluorescence intensity value related to the median fluorescence intensity of these antigens on B-, T-, and NK-lymphocytes, respectively (a representative example of CD38 expression is shown in the Figure $2 \mathrm{~b}, \mathrm{~A}, \mathrm{~B})$. This approach enables to quantify the expression of a given antigen as higher $(+) \uparrow$ or weaker $(+) \downarrow$ in Burkitt-like lymphoma with 11q aberration and in Burkitt lymphoma cells than in control lymphocytes, as well as to compare the expression of pan-B antigens in Burkittlike lymphoma with 11q aberration and Burkitt lymphoma cells (ie, CD19 vs CD20 vs CD22, using monoclonal antibodies conjugated with the same fluorochrome), as described.1,4,12 'Dim' (lower and heterogeneous) or 'bright' (higher and homogeneous) expression was defined as previously. ${ }^{4,12}$ Simultaneously, cytological smears were stained with a hematoxylin-eosin and May-Grünwald-Giemsa for morphological evaluation (Figure 2a).

\section{Conventional Cytogenetics and FISH}

For conventional cytogenetics, fresh fine needle aspiration biopsy samples were fixed directly or following standard cultures.,4,12 Karyotypes were classified according to the ISCN $2016 .{ }^{13}$ To study the presence of MYC/BCL2/BCL6 translocations (MYC/ BCL2/BCL6 break apart probes) and to assess the number of copies, precise positions, possible inversions of $C C N D 1 / A T M / K M T 2 A$ loci and terminal $11 \mathrm{q}$ deletions (CCND1/ATM/KMT2A/D11S1037/CEP11 locus-specific probes), FISH was performed using commercial probes (Vysis Abbott Molecular, Downers, Grove, IL, USA), as previously described. ${ }^{1,2,4}$ Representative karyotype and FISH results are shown in Figure 4.

\section{Copy-number Analysis}

Copy number and copy-number-neutral loss of heterozygosity were analyzed in Burkitt-like lymphoma with 11q aberration and Burkitt lymphoma carrying both $M Y C$ rearrangement and $11 \mathrm{q}$ aberration cases using CytoSure Haematological Cancer and SNP Array $(8 \times 60 \mathrm{k})$ (Oxford Gene Technology (OGT), Yarnton, UK), as previosuly described. ${ }^{14}$ Copy-number data have been deposited at the Gene Expression Omnibus (GEO) database (GSE93002).

\section{Statistics}

Fisher's exact test was used to measure associations between categorical variables using $\mathrm{R}$ software (3.2.3 version). $P$-values $<0.05$, adjusted using the Benjamini-Hochberg method, were considered as statistically significant. Overall Survival was estimated by the Kaplan-Meier method (STATISTICA v.9.1, Statsoft).

\section{Results}

Morphologically, all cases of Burkitt-like lymphoma with $11 \mathrm{q}$ aberration showed a diffuse lymphoid infiltration, 4 exhibited a classical starry-sky pattern, typical of Burkitt lymphoma (Figure 2a), while the other 6 slightly differed from classical Burkitt lymphoma features by the reduced number of macrophages and apoptotic bodies. In most tumors, the cell size was uniformly medium, with round nuclei and a few small nucleoli, typical of Burkitt lymphoma. However, the lack of a jigsaw puzzle effect of cytoplasmic borders and a mild degree of irregular nuclear contours were noted in some tumors (Supplementary Table 3).

The immunohistochemistry of Burkitt-like lymphoma with 11q aberration was characteristic of Burkitt lymphoma. All 10 cases expressed a homogeneous phenotype of germinal center origin (CD10 +/BCL6+/MUM1 -/usually CD44-), with a high Ki-67 proliferation index, always $>95 \%$ (100\% and 

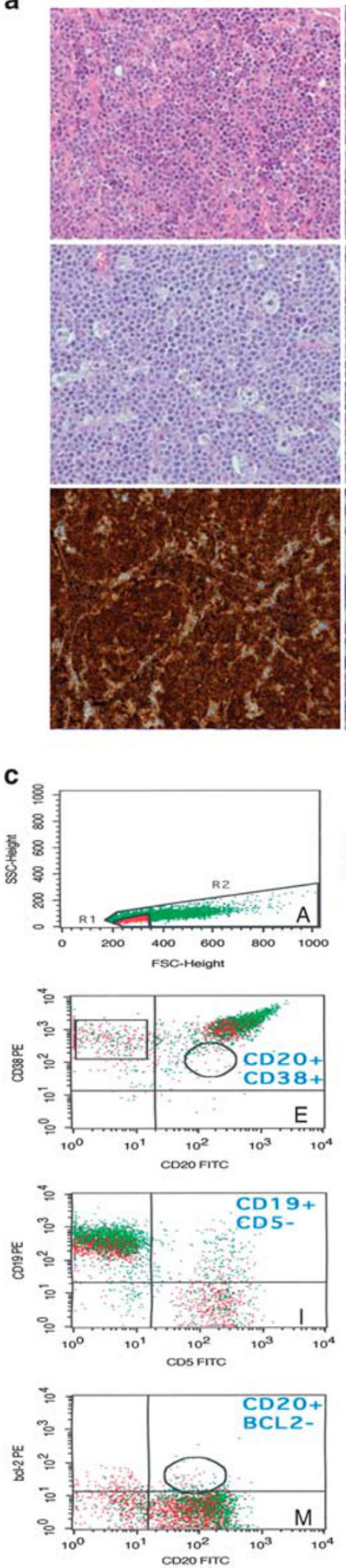
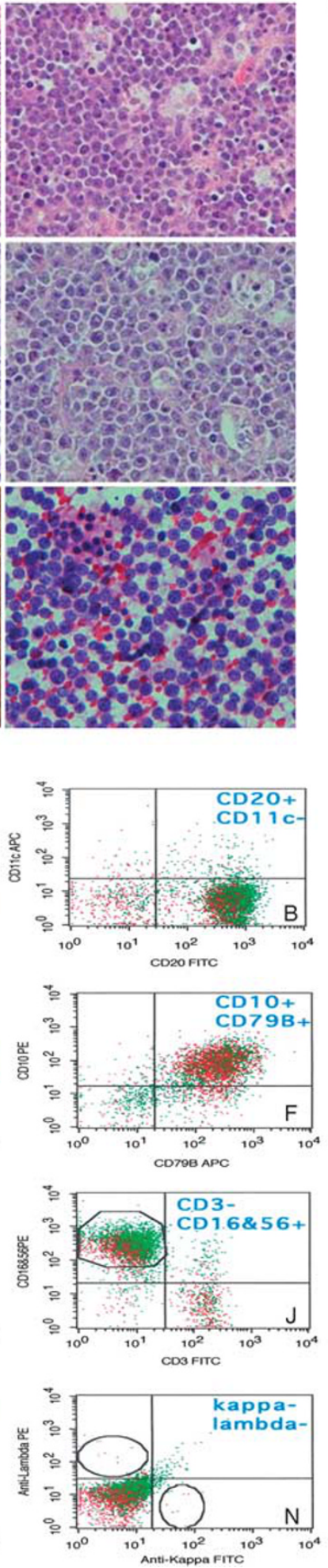

b
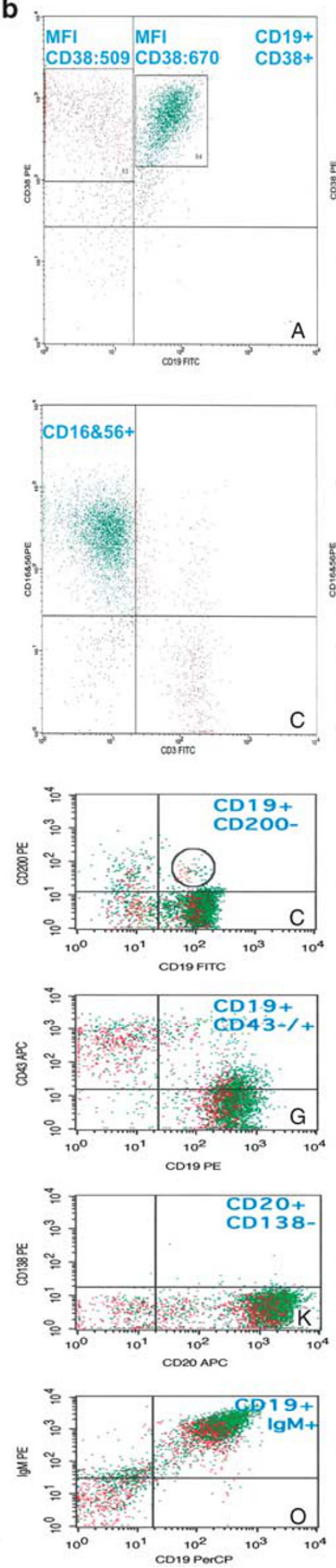
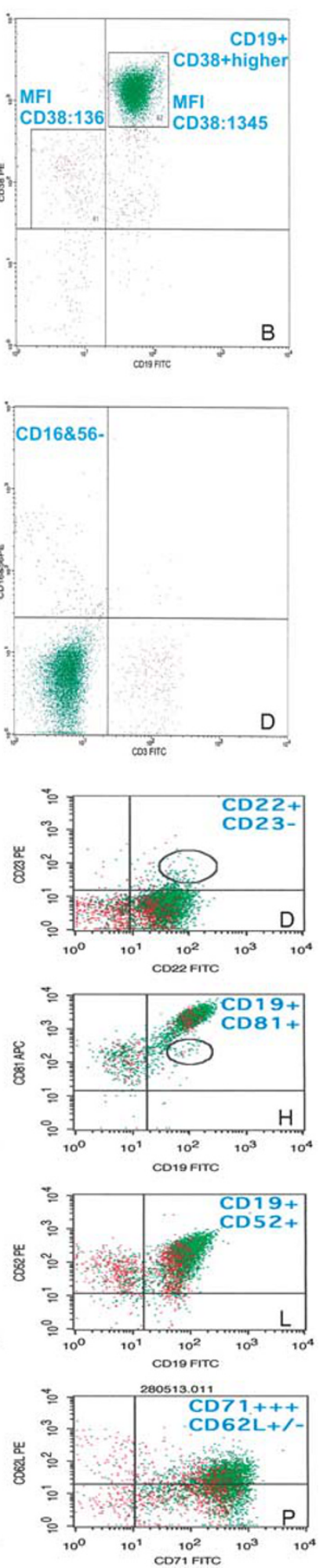

$>95 \%$ in 8 and 2 cases, respectively) and were negative for BCL2/MUM1/cyclin D1/CD3/CD5/TdT/ EBER expression. By immunohistochemistry, these tumors expressed LMO2/CD43/CD44 in 70\%/30\%/ $20 \%$ of cases, respectively (Supplementary Table 3). The two examined cases with Burkitt lymphoma

carrying both $M Y C$ rearrangement and $11 \mathrm{q}$ aberration were negative for LMO2. Independent of MYC status at gene level, the percentage of MYCexpressing cells (monoclonal antibody clone Y69) exceeded the 2016 WHO threshold of $40 \%$ of immunohistochemical positivity. ${ }^{5}$ A strong CD56 
Figure 2 Pathomorphological diagnosis of Burkitt-like lymphoma with 11q aberration. (a) Histopathological, immunohistochemical, and cytopathological features of Burkitt-like lymphoma with 11q aberration. Classical Burkitt lymphoma histopathology of Burkitt-like lymphoma with 11q aberration cases no.1 (upper row) and 9 (middle row), a cytopathology (obtained by fine needle aspiration biopsy of abdominal tumor) of case 9, and CD56 positive immunohistochemical reaction of case no.8 (lower row). Diffuse growth is composed of medium-sized lymphoid cells showing jigsaw puzzle effect of cytoplasmic borders with a starry pattern due to admixed phagocytic macrophages. The nuclei are similar in size and shape (paraffin section stained with hematoxylin and eosin). Upper and intermediate panels, original magnification $\times 200$ (left panel) and $\times 400$ (right panel); in cytopathological smears and immunohistochemical reaction, original magnification $\times 400$ (lower row); (b) flow-cytometry-based analysis of median fluorescence intensity of CD38 expression (A, B) and CD16/CD56 (C, D) in Burkitt-like lymphoma with 11q aberration and Burkitt lymphoma. Median fluorescence intensity (MFI) of CD38 expression on Burkitt-like lymphoma with 11q aberrations is similar to normal T lymphocyte expression - CD38(+) (plot A), and in Burkitt lymphoma it is higher - CD38(+)higher (plot B). The absence of CD38higher (plot A) and CD16/CD56+ (plot C) characterized Burkitt-like lymphoma with 11q aberration. CD38higher (plot B) and the lack of CD16/CD56 (plot D) characterized Burkitt lymphoma; (c) fine needle aspiration biopsy/flow cytometry analysis of Burkitt-like lymphoma with 11q aberration (case 9). Forward scatter/side scatter dot plots presenting both small normal T/B-lymphocytes (red cells) and larger lymphoma cells (green cells). Burkitt-like lymphoma with 11q aberration express: CD20/CD19/CD22 (median fluorescence intensity CD20 > CD19 > CD22)/CD38/CD10/CD79/CD81higher/CD52/IgM/ CD16/CD56/CD71 as well as CD43/CD62L of weak intensity, on a small subpopulation of cells, and are negative for CD5/CD11c/CD23/ CD138/CD200/BCL2/ $\kappa$ and $\lambda$. Plots E and J show a similar level of CD38 expression in comparison to normal T-lymphocytes CD38 (in the box) and CD16/CD56+ (in the box) on 'Burkitt-like lymphoma with 11q aberration' cells. Antigen expression of a small number of normal B-lymphocytes is marked by circles.

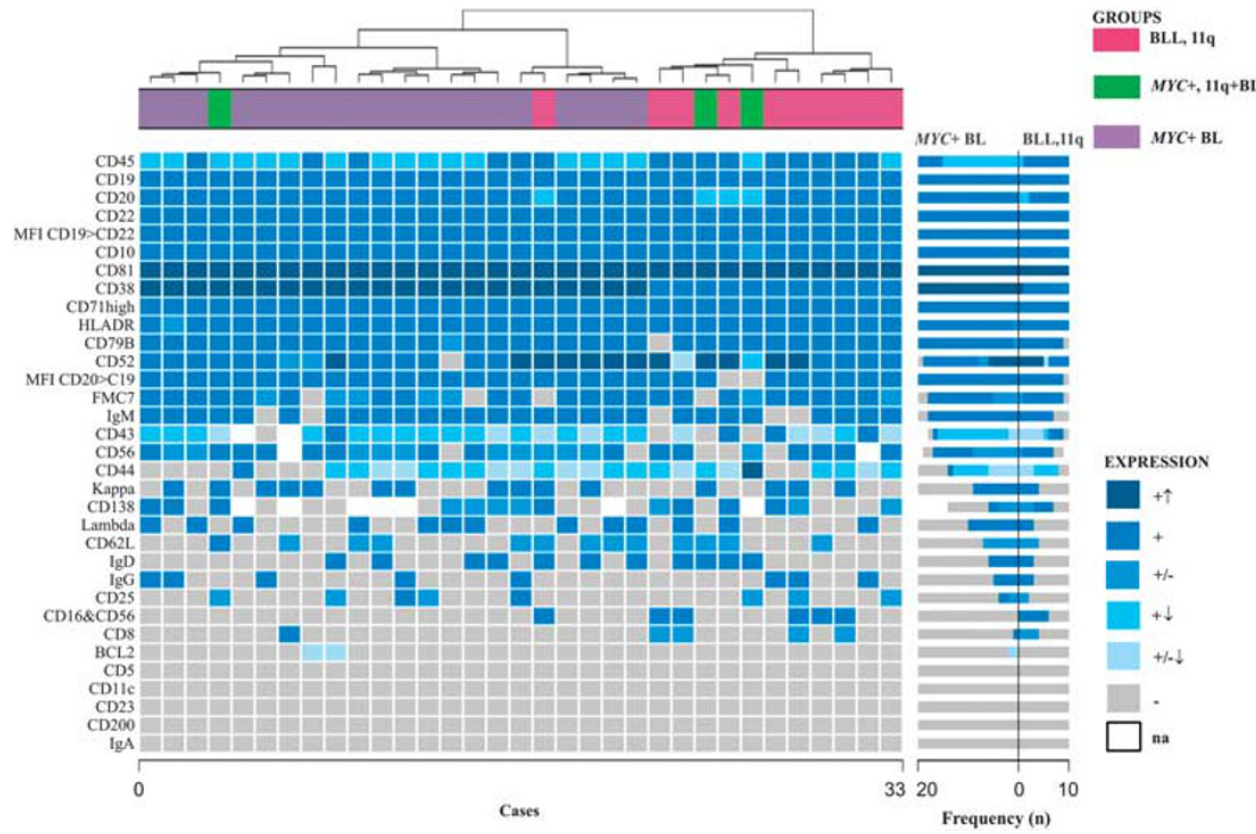

Figure 3 Cluster (variables and cases) analysis of 10 Burkitt-like lymphomas with 11q aberration (BLL, 11q), vs 23 MYC-positive Burkitt lymphomas $\left(M Y C^{+} \mathrm{BL}\right)$, including three cases with an additional 11q aberration $\left(M Y C^{+}, 11 \mathrm{q}^{+} \mathrm{BL}\right)$. Expression: $(+) \uparrow$, an antigen with higher expression in Burkitt-like lymphoma with 11q aberration cells compared to normal B/T/NK-lymphocytes in $100 \%$ of cells; (+), positive in $100 \%$ of cells; $(+) \downarrow$, an antigen with weaker expression in Burkitt-like lymphoma with 11q aberration cells compared to normal B/T/NKlymphocytes in $100 \%$ of cells; (+/-), positive in $>20 \%$ to $<100 \%$ of cells; $(+/-) \downarrow$, an antigen with weaker expression in Burkitt-like lymphoma with 11q aberration cells compared to normal B/T/NK-lymphocytes in $>20 \%$ to < $100 \%$ of cells; (-), no expression (i.e. expression in $<20 \%$ cells); nd, not done.

staining was found in $30 \%$ and $40 \%$ of cases of Burkitt-like lymphoma with $11 \mathrm{q}$ aberration, using mouse and rabbit monoclonal antibodies, respectively.

In the majority of Burkitt-like lymphomas with 11q aberration, flow cytometry analysis showed $>85 \%$ of lymphoma cells and few normal T-lymphocytes. Forward and side scatter dot-plot showed two distinct cell clusters, small T/B-lymphocytes and slightly larger, homogenous neoplastic cells (Figure 2c). All cases were CD45/CD19/CD20/ CD22/FMC7/CD81 higher/BCL6/CD10/CD52/HLA-DRpositive with median fluorescence intensity of CD19 higher than that of CD22, while negative for CD5/
CD11c/CD23/CD200/BCL2. The median fluorescence intensity of CD20 expression was higher than that of CD19 in 90\% of Burkitt-like lymphomas with $11 \mathrm{q}$ aberration and $100 \%$ of $M Y C$-positive Burkitt lymphoma cases. The diminished expression of CD45 was less frequent in Burkitt-like lymphoma with $11 \mathrm{q}$ aberration than in $M Y C$-positive Burkitt lymphoma ( $10 \%$ vs $74 \%$, adjusted $P=0.0077)$, while CD38higher expression ${ }^{4,15-17}$ (CD38, PE-conjugated HB7 clone, Figure 2b, A, B) was more frequent in MYC-positive Burkitt lymphoma than in Burkitt-like lymphoma with $11 \mathrm{q}$ aberration $(91 \%$ vs $10 \%$, adjusted $P<0.001$ ). Two out of 3 cases of Burkitt lymphoma carrying both $M Y C$ rearrangement and 
a

b normal aberrant

chromosome $11 \quad$ chromosome 11

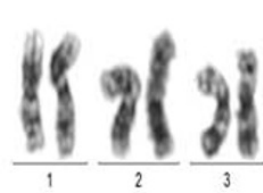

in

Q 15 - $\square_{15}$
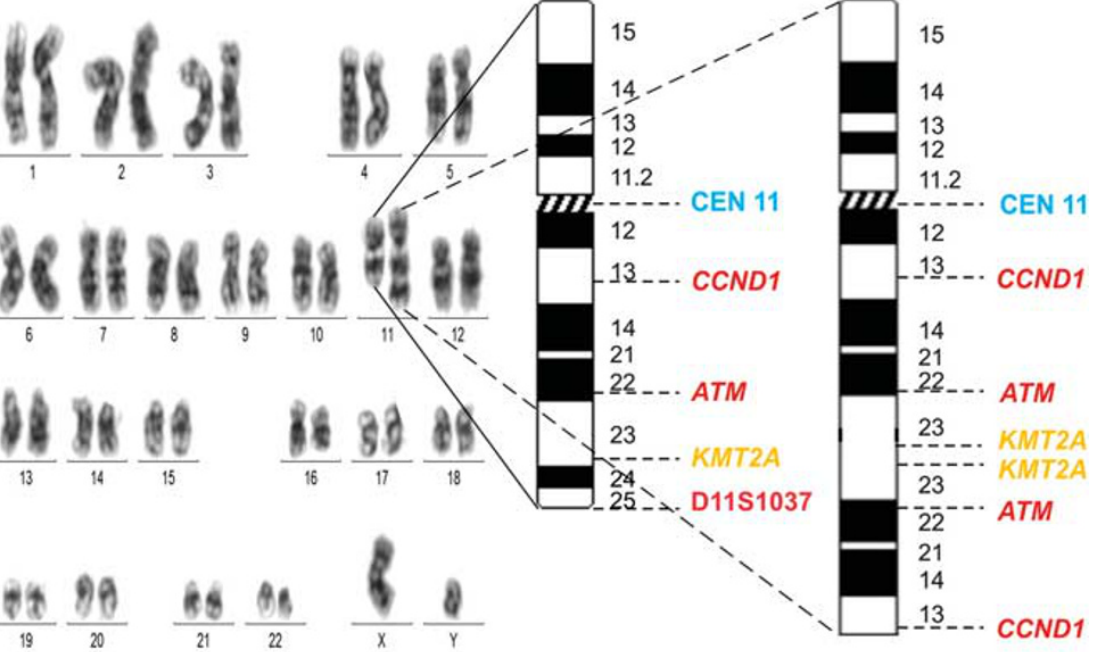

C
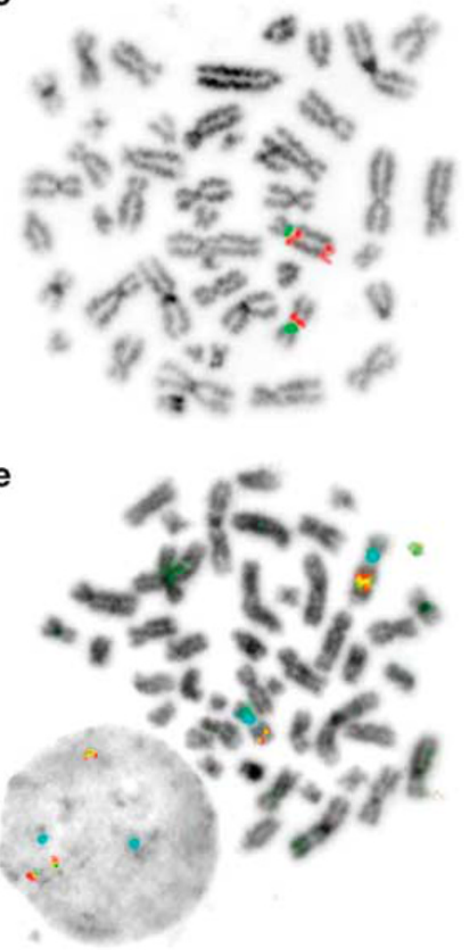

d

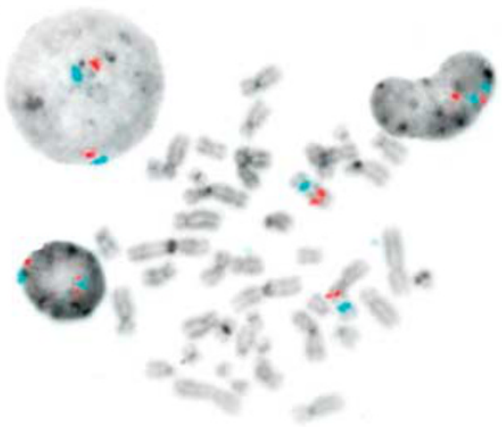

f

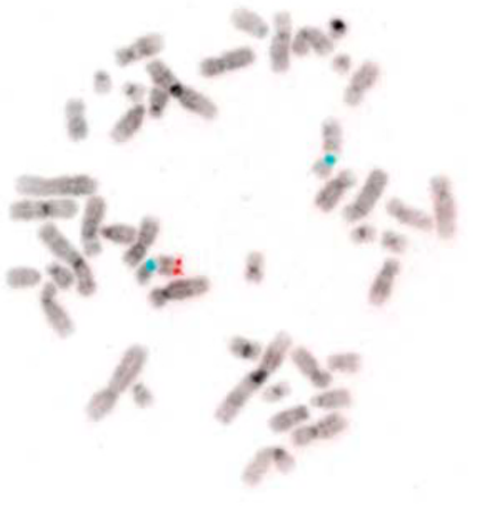

Figure 4 Conventional cytogenetic diagnosis of Burkitt-like lymphomas with 11q aberration. (a) Simple karyotype with 11q aberration, 46, XY,dup(11)(q23.3q13.1), as a sole abnormality in case no.1; (b) FISH probe positions on normal chromosome 11 and on aberrant chromosome 11, with duplication and inversion of 11q, which explains the results shown on the subsequent FISH analysis; (c-f) FISH analysis with chromosome 11 specific probes on metaphases. Each metaphase shows one normal copy of chromosome 11 and one copy of 11q aberration; (c) CEP-11 Spectrum Green, CCND1 Spectrum Orange. Big distance between two red signals in 11q aberration indicates an inversion of duplicated region (case no.4); (d) CEP-11 Spectrum Aqua, ATM Spectrum Orange. Small distance between two red signals in $11 \mathrm{q}$ aberration designates an inversion of the duplicated region. A similar pattern of signals is visible in interphase nucleus (case no.9); (e) CEP-11 Spectrum Aqua, KMT2A BAP. Colocalization and multiplication of red-green signals point to 11q inversion with multiplication of the region harboring KMT2A. A similar signal pattern is visible in interphase nucleus (case no.9); (f) CEP-11 Spectrum Aqua, D11S1037 Spectrum Orange. One red 11q telomeric signal indicates normal chromosome 11, no red signal in 11q aberration confirms terminal deletion (case no.4).

11q aberration also showed CD38 ${ }^{\text {higher }}$ expression. Other markers in Burkitt-like lymphoma with 11q aberration were expressed as follows: $\operatorname{CD} 79 \beta(90 \%)$, CD43 (90\%), CD44 (80\%, but expression in each case was always weaker than on T lymphocytes), CD56 (78\%), CD16/CD56(60\%), CD138(70\%), CD62L $(40 \%$, but usually as a weak expression in slightly over $20 \%$ of tumor cells, Figure 2c, P), CD25(20\%) 
and CD8 ${ }^{\text {weak }}(40 \%)$, with a diverse intensity (Figure 3, Supplementary Table 4). The expression of all those antigens was similar in $M Y C$-positive Burkitt lymphoma and Burkitt-like lymphoma with 11q aberration, except for CD16/CD56 (containing PE-labeled CD16, clone B73.17-9, and PE-labeled CD56, clone MY31,10, Figure 2b, C, D) and CD8 that were not expressed on MYC-positive Burkitt lymphoma (0\% vs 60\% cases, adjusted $P=0.0019$, and $4 \%$ vs $40 \%$ cases, adjusted $P=0.018$, respectively), as well as CD43, expressed in all $(+,+\downarrow)$ Burkitt lymphoma cells $(71.4 \%$ vs $40 \%$ cases, adjusted $P=0.0178)$. In addition, CD71 (+++) expression, representing proliferative activity, was always detected in $100 \%$ of cells in both Burkitt-like lymphoma with 11q aberration and Burkitt lymphoma. Monoclonal light chain expression of moderate intensity was found in $90 \%$, and no $\kappa / \lambda$ expression in $10 \%$ of cases. IgH (monoclonal heavy immunoglobulin chain) expression included: IgD $(+) / \operatorname{IgM}(+)$ in $30 \%, \operatorname{IgM}(+) / \operatorname{IgG}(+)$ in $10 \%, \operatorname{IgG}(+)$ in $20 \%, \operatorname{IgM}(+)$ in $30 \%$, and $\operatorname{IgH}(-)$ in $10 \%$ of cases. Detailed flow cytometry characteristics of Burkittlike lymphoma with 11q aberration and Burkitt lymphoma are shown in Supplementary Tables 4 and 5 , respectively.

Copy-number analysis was performed, if fresh material was available, ie, in nine cases of Burkittlike lymphoma with 11q aberration (Figure 5a) and two of Burkitt lymphoma carrying both MYC rearrangement and $11 \mathrm{q}$ aberration (Figures $5 \mathrm{a}$ and b). Both groups showed similar levels of genetic complexity with the mean number of 5.5 alterations per case, as previously described, ${ }^{2}$ and usually a simple (Figure 4a) or low complex karyotype. Nine out of 10 cases of Burkitt-like lymphoma with 11q aberration showed a recurrent pattern of gain/loss (Figures 5a and b), consistent with the copy-number profile of cases 1-3 previously analyzed by Agilent $244 \mathrm{k}$ array. $^{2}$ In case 10 , a $774 \mathrm{~Kb}$ homozygous deletion at 11q24.3 was detected. This alteration targeted ETS1, FLI1, KCNJ1, KCNJ5, C11orf45 and TP53AIP1 (Figure 5b). In case 8, 11q duplication was not accompanied by a terminal deletion, but the analysis of single-nucleotide polymorphisms revealed a stretch of copy-number neutral loss of heterozygosity at 11q24.1-q25 (Figure 5c). As shown by FISH, the duplicated area in all cases comprised an inversion of the fragment that was gained, with a terminal deletion of 11q (Figures $4 \mathrm{~b}-\mathrm{f}$ and $5 \mathrm{a}$ ).

\section{Discussion}

We characterized 10 cases of Burkitt-like lymphoma with 11q aberration identified among adult BL patients (10 out of $82,12 \%$ ), originally diagnosed and treated as MYC-negative Burkitt lymphoma at a single institution. Salaverria et $a l,{ }^{2}$ in a series including both children and adults, identified $3 \%$ of all molecularly defined Burkitt lymphoma to be Burkitt-like lymphoma with 11q aberration.

Burkitt-like lymphoma with 11q aberration shows a number of clinicopathological and molecular similarities to $M Y C$-positive Burkitt lymphoma. ${ }^{1-4,18}$ Typical of Burkitt-like lymphoma with 11q aberration, as we have shown here and previously, ${ }^{1,2,4}$ was one peak incidence in HIV/EBV-negative young adult males. The typical presentation, predominantly nodal/tonsilar, was a single bulky lymph node/tumor, or less frequently, with a few contiguous lymph nodes/tumors involved, without bone marrow and central nervous system involvement, while in the Salaverria series, ${ }^{2}$ there were children with bone marrow involvement. This may be due to lack or very weak expression of CD62L (L-selectin) on a small subpopulation of cells of adult Burkittlike lymphoma with 11q aberration. CD62L is a cell adhesion molecule, playing an important role in lymphoma dissemination. ${ }^{19}$

A cohort of patients with Burkitt-like lymphoma with 11q aberration had a similar relapse-free outcome to that in patients with $M Y C$-positive Burkitt lymphoma, if treated with Burkitt lymphomadirected regimen, ${ }^{10,11,20}$ in contrast to patients with Burkitt-like lymphoma with $11 \mathrm{q}$ aberration treated with R-CHOP (Rituximab, cyclophosphamide, doxorubicin, vincristine, and prednisone), who tend to relapse, ${ }^{21}$ like one of our patients, originally misdiagnosed as diffuse large B-cell lymphoma and treated accordingly at another hospital. By now, 10 patients presented here achieved a sustained complete remission, and 8 are still alive. In the abovementioned retrospective study by Sevilla et $a l,{ }^{21}$ adults with $M Y C$-positive Burkitt lymphoma had longer survival than patients with $M Y C$-negative Burkitt lymphoma, but were treated according to $M Y C$ status, and most of the adult $M Y C^{-}$patients (that could include the currently recognized Burkittlike lymphoma with $11 \mathrm{q}$ aberration entity) received the designed for diffuse large B-cell lymphoma regimens, that seem suboptimal to cure Burkitt-like lymphoma with $11 \mathrm{q}$ aberration. Five-year overall survival of patients in our series of Burkitt-like lymphoma with $11 \mathrm{q}$ aberration was $80 \%$, similar to that of adult $M Y C$-positive Burkitt lymphoma. ${ }^{20}$ Nevertheless, after initial excess of non-relapse mortality, the survival curves of the patients with Burkitt-like lymphoma with 11q aberration reached a plateau, which is typical of $M Y C$-positive Burkitt lymphoma. ${ }^{10,11,20}$

Tumors of Burkitt-like lymphoma with 11q aberration may have diverse morphology, mainly of Burkitt lymphoma, sometimes of B-cell lymphoma unclassifiable with features intermediate between diffuse large B-cell lymphoma and Burkitt lymphoma, and sporadically of diffuse large B-cell lymphoma. ${ }^{4,5}$ As we show here, Burkitt-like lymphoma with 11q aberration have immunophenotype characteristics by immunohistochemistry similar to $M Y C$-positive Burkitt lymphoma, with the exception of CD43, 


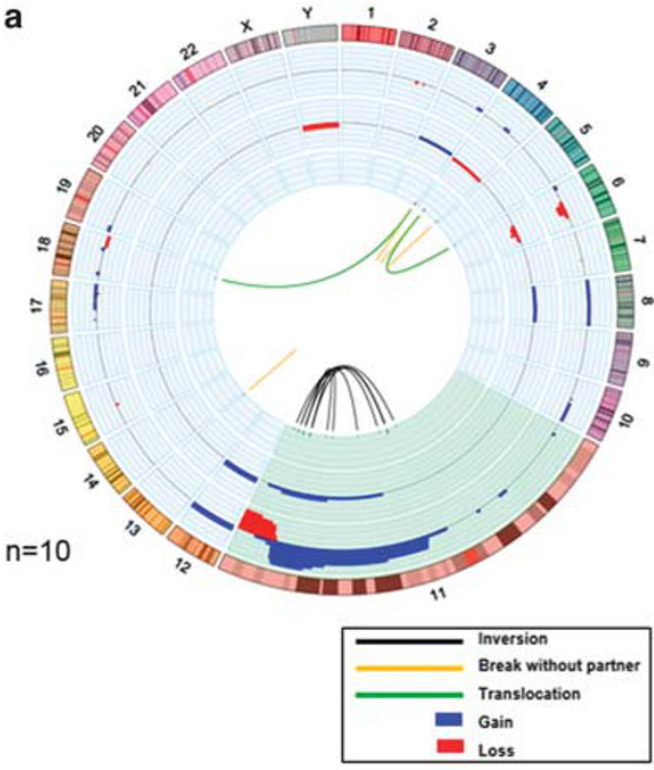

C

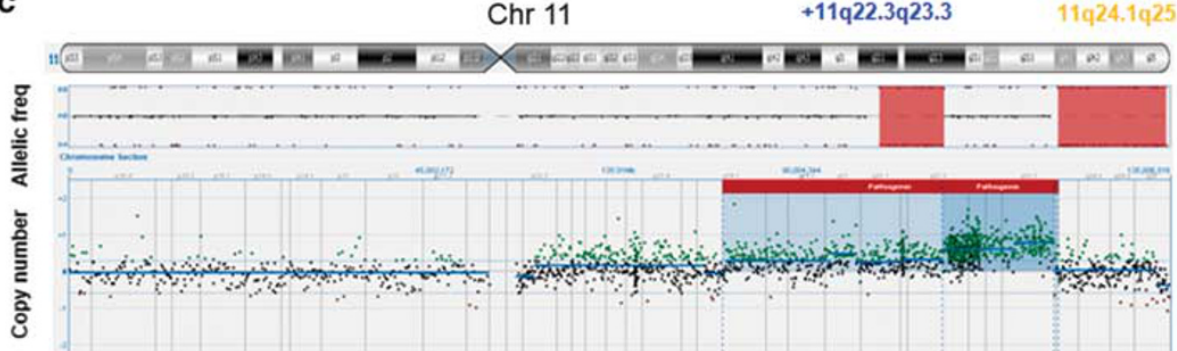

b

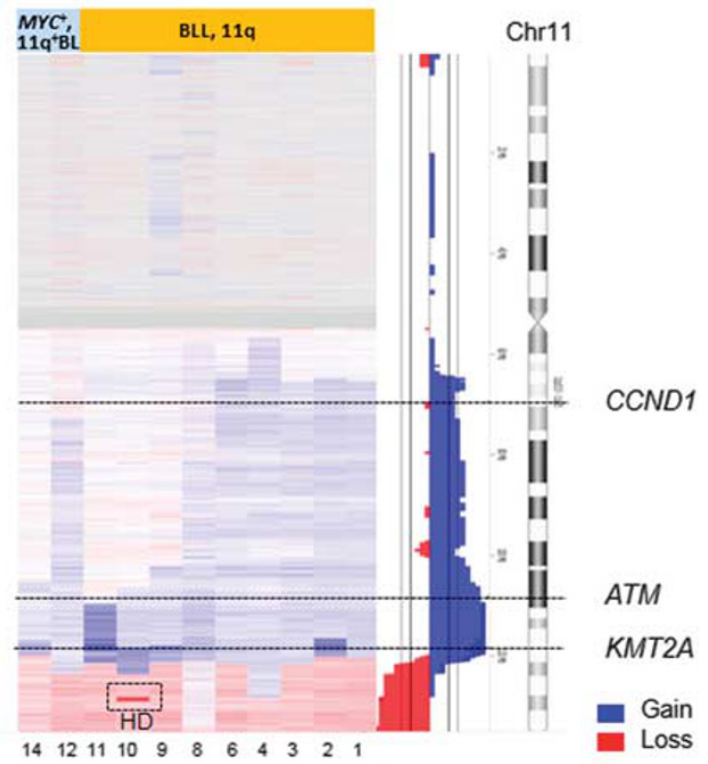

- GAIN

Stretch of $\mathrm{LOH}$

Figure 5 Molecular and cytogenetic analysis of Burkitt-like lymphomas with 11q aberration. (a) Circular representation of copy number and structural variants in 10 Burkitt-like lymphoma with 11q aberration cases. Chromosomes are represented in the outer layer, the percentage of regions of loss (red) and gain (blue) are shown in the inner layers (outer layer, CN array data, $n=9$, and inner layer, karyotype data, $n=10$ ). The internal arcs represent interchromosomal translocations detected by conventional cytogenetics and are marked black (inversion), green (translocations) and orange (break without partner); (b) a view of chromosome 11 analyzed by copy-number array $(8 \times 60 \mathrm{k})$ in 9 Burkitt-like lymphomas with 11q aberration (BLL, 11q) and 2 cases of Burkitt lymphoma carrying both $M Y C$ rearrangement and $11 \mathrm{q}$ aberration $\left(\mathrm{MYC}^{+}, 11 \mathrm{q}^{+} \mathrm{BL}\right)$; HD, a $774 \mathrm{~Kb}$ homozygous deletion at 11q24.3 in case 10; (c) 11q aberration pattern in case 8, dot plots present large 11q duplication with amplification region, without terminal deletion. In the allelic frequency diagram, the dark red block indicates a stretch of copy-number neutral loss of heterozygosity at 11q24.1q25.

LMO2, and CD56 expression. In this study, LMO2 was expressed in $70 \%$ of cases of Burkitt-like lymphoma with $11 \mathrm{q}$ aberration and in none of Burkitt lymphoma carrying both $M Y C$ rearrangement and $11 \mathrm{q}$ aberration. Colomo et $a{ }^{9}{ }^{9}$ in a series including molecularly defined BL, identified 45/46 (98\%) MYC-positive Burkitt lymphoma cases as LMO2 negative. Taken together, these findings confirm that LMO2 expression may be a good predictor of $M Y C$ translocation, and may contribute to differential diagnosis of Burkitt lymphoma. The lack of LMO2 expression is typical of $M Y C$-positive Burkitt lymphoma and LMO2 is usually expressed in Burkitt-like lymphoma with 11q aberration.

We have previously described flow cytometry immunophenotype of Burkitt lymphoma. ${ }^{18,20,22}$ In the current study, antigen expression patterns in all Burkitt-like lymphomas with 11q aberration are similar to that of Burkitt lymphoma, but with some statistically significant differences. First, we found
CD16/CD56 expression in 60\% of Burkitt-like lymphomas with $11 \mathrm{q}$ aberration and in none of Burkitt lymphomas carrying both $M Y C$ rearrangement and 11q aberration, or $M Y C$-positive Burkitt lymphoma. CD16/CD56 is an indicator of NK differentiation, but CD16/CD56 antibody is not currently available for immunohistochemistry. There are two types of CD16, CD16A, which is a transmembrane protein found on NK cells, and CD16B found on neutrophils. Most CD56 bright $\mathrm{NK}$ cells in the peripheral blood express little to no CD16A. In contrast, the majority of CD56 $6^{\text {dim }}$ cells uniformly express high levels of CD16A, ${ }^{23}$ detectable by CD16/CD56 antibody. By flow cytometry, we identified CD56 expression in $78 \%$ of Burkitt-like lymphomas with 11q aberration and $89 \%$ of $M Y C$-positive Burkitt lymphomas. Interestingly, a proportion of Burkitt-like lymphomas with 11q aberration, but none of Burkitt lymphomas, also expressed neural cell adhesion molecule (CD56) by immunohistochemistry, which indicates that it 
would be desirable to include immunohistochemistry for CD56 along with LMO2, ${ }^{9}$ in the extended diagnostic panel for Burkitt-like lymphoma with 11q aberration and Burkitt lymphoma. CD56 expression is an unusual feature in B-cell lymphomas, with 0.5 to $5.5 \%$ expression rate. ${ }^{24}$ Therefore, commercially available CD16/CD56 antibody mixture for flow cytometry (containing PE-labeled CD56, clone MY31,10, which seems to be specific for $11 \mathrm{q}$ aberration-data not shown) currently presents the best sensitivity for the detection of $11 \mathrm{q}$ aberration. Second, we found a significant difference in CD45 expression between Burkitt-like lymphoma with 11q aberration and Burkitt lymphoma, with a decreased expression of this antigen in Burkitt lymphoma. Of note, CD16A (FCGR3A) and CD45 (PTPRC) loci, the 1q23 and 1q31.3-q32.1, are typically gained in $M Y C$ positive Burkitt lymphoma, ${ }^{25}$ whereas $C D 56$ (NCAM1) lies at 11q23, the region where duplication $11 \mathrm{q}$ takes place in most cases of Burkitt-like lymphoma with $11 \mathrm{q}$ aberration. ${ }^{1,2}$ It cannot be excluded that differences in CD16/CD56 and CD45 expression between Burkitt-like lymphoma with 11q aberration and Burkitt lymphoma result from duplication of the above-mentioned regions. As shown by CD71 expression in flow cytometry and by Ki-67 index in immunohistochemistry, all cells of Burkittlike lymphoma with 11q aberration presented high proliferative activity, typical of Burkitt lymphoma. ${ }^{26}$ Notably, there is another ontogenetic link between Burkitt-like lymphoma with 11q aberration and Burkitt lymphoma, i.e. heavy immunoglobulin chain cell surface expression pattern that in Burkitt-like lymphoma with $11 \mathrm{q}$ aberration is most commonly $\operatorname{IgD}(+) / \operatorname{IgM}(+)$, followed by $\operatorname{IgM}(+)$, while in Burkitt lymphoma most frequent $\operatorname{IgM}(+)$ is followed by $\operatorname{IgD}$ $(+) / \operatorname{IgM}(+)$. Third, we demonstrated the absence of CD38 higher expression in Burkitt-like lymphoma with 11q aberration (where CD38 expression comparable to that on T lymphocytes was seen), while CD38higher was typical of Burkitt lymphoma. Therefore, detection of CD16/CD56 ${ }^{+}$and the absence of CD38higher expression by means of fine needle aspiration biopsy/flow cytometry procedure, completed within $1.5 \mathrm{~h}$, appears to be a reliable, fast, easy, and costeffective method for diagnosing $11 \mathrm{q}$ aberration. Importantly, we show that flow cytometric CD38 ${ }^{\text {higher }}$ expression, consistently present in $M Y C$ positive lymphomas, ${ }^{4,12,15-17,22,27}$ is a more reliable and quickly detectable indicator of $M Y C$ translocation in $\mathrm{CD} 10(+)$ aggressive lymphomas, ${ }^{15,16}$ compared to MYC immunohistochemical staining, since MYC expression is highly variable, and not always correlates with $M Y C$ rearrangements. ${ }^{3,5,28}$ Moreover, a recent study that compared LMO2 with MYC protein staining by immunohistochemistry showed that the lack of LMO2 expression is a better surrogate of $M Y C$ translocation status than the immunohistochemical MYC expression, indicating an advantage for LMO2 immunohistochemistry, particularly in the CD10(+) subgroup. ${ }^{9}$

Compared to histopathology and immunohistochemistry, flow cytometry allows for a closer examination of tumor heterogeneity and identification of subtle differences with regard to antigen expression. Also, fine needle aspiration biopsy proved to be highly efficient way of obtaining tumor cells for karyotype, FISH, and molecular studies. Characteristic $11 \mathrm{q}$ aberrations, predominantly dup (11)(q23q13), is visualized by karyotyping. Based on our experience with routine histopathology and immunohistochemical examination performed simultaneously with fine needle aspiration biopsy/ flow cytometry/conventional cytogenetics/FISH, we postulate that in specialized hematology-oncology centers, combination of these two diagnostic

Table 2 Flow cytometry/immunohistochemistry-based approach to differential diagnosis of Burkitt lymphoma vs Burkitt-like lymphoma with $11 \mathrm{q}$ aberration

Burkitt lymphoma

Burkitt-like lymphoma with $11 q$ aberration

Antigen expression by flow cytometry in fine needle aspiration biopsy samples

CD45(+) weaker/CD38(+) $)^{\text {higher/ CD16/CD56(-) /CD8(-) /CD43(+) or* }}$ CD45(+) ${ }^{\text {bright/CD38(+)/CD16/CD56(+) or* CD16/CD56(-) /CD8(+) or* }}$ CD43(+/-),

MFI CD20 $(+)^{\text {bright }}>$ MFI CD19 $(+)^{\text {brigh t }}>$ MFI CD22(+),

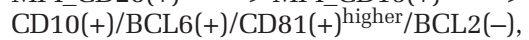

CD44(+ ) or* CD44(+/-) or* CD44(-),

CD56(+/-) or* CD56(+) or* CD56(-)/ CD138(-) or* CD138(+/-) or* CD138(+),

CD79 $\beta(+) / F M C 7(+)$ or* FMC7(+/-) or* FMC7(-)/ג(+) or* $\kappa(+)$, $\operatorname{IgM}(+)$ or ${ }^{*} \operatorname{IgM}(+) / \operatorname{IgD}(+)$ or ${ }^{*} \operatorname{IgM}(+) / \operatorname{IgG}(+)$ or ${ }^{*} \operatorname{IgG}(+)$ or ${ }^{*} \operatorname{IgH}(-)$, CD5(-)/ CD11c(-)/CD23/(-)/CD25(-) or* CD25(+/-)/CD62L(-) or* CD62L(+/-)/ HLADR(+)/CD200(-) CD52(+), $100 \%$ of cells positive for CD71(+++).

CD8(-)/ CD43(+/-) or* CD43(+) or* CD43(-),

MFICD20 $(+)^{\text {bright }}>$ MFI CD19(+) ${ }^{\text {bright }}>$ MFI CD22(+),

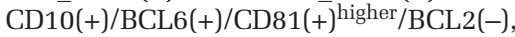

CD44(+\) or* CD44(+/-) or* CD44(-),

CD56(+) or* CD56(-) or* CD56(+/-)/ CD138(+) or* CD138(+/-) or* CD138(-)

CD79 $\beta(+) / F M C 7(+) / \kappa(+)$ or* $\lambda(+)$,

$\operatorname{IgM}(+) / \operatorname{IgD}(+)$ or* $\operatorname{IgM}(+)$ or* $\operatorname{IgG}(+)$ or* $\operatorname{IgM}(+) / \operatorname{IgG}(+)$ or* $\operatorname{IgH}(-)$, CD5(-)/CD11C(-)/CD23(-)/CD25(-) or* CD25(+/-)/CD62L(-) or* CD62L(+/-)/ HLADR(+)/ CD200(-) CD52(+),

$100 \%$ of cells positive for CD71(+++)

Antigen reaction by immunohistochemistry $\mathrm{LMO} 2(-)^{\wedge} / \mathrm{CD} 56(-) / \mathrm{CD} 43(+)^{\wedge}$

LMO2(+)^/CD56(+/-)\#/CD43(+/-)\#

Bold letters mark significant statistical differences between Burkitt-like lymphoma with 11q aberration and typical $M Y C$-positive Burkitt lymphoma, which may be useful in the differential diagnosis; *alternative expressions are ordered from more to less frequent; MFI: median flourescence intensity; immunohistochemical staining: ^usually negative or positive; (+/-)\#sometimes positive. 
approaches is a reliable method for credible diagnosis of Burkitt-like lymphoma with 11q aberration and Burkitt lymphoma. Considering these results, as well as previous data, $, 18,20,22$ we propose a practical flow cytometry and immunohistochemistry-based approach to the diagnosis of Burkitt-like lymphoma with $11 \mathrm{q}$ aberration and Burkitt lymphoma, as summarized in Table 2.

In the current study, we confirm a pattern of an inverted duplication of a part of the long arm of chromosome $11^{1}$ with mono- or biallelic telomeric loss of $11 \mathrm{q},{ }^{2,4}$ which we have previously referred to as a critical set of $11 \mathrm{q}$ aberrations, ${ }^{18}$ as a recurrent $11 \mathrm{q}$ abnormality. One case that presented no 11q deletion detected by single-nucleotide polymorphism analysis/array-comparative genomic hybridization and FISH showed a uniparental disomy in the critical 11q24.1q25 deletion region. We find the telomeric stretch of copy-number neutral loss of heterozygosity (often referred to as uniparental disomy) in this case, to be an alternative genetic event to the terminal deletion, possibly resulting in an equivalent molecular effect. Inverted duplication of $11 \mathrm{q}$ is not included in the latest update of 2016 WHO classification, ${ }^{5}$ still we found the inversion in all cases with Burkitt-like lymphoma with 11q aberration.

In conclusion, we show significant differences between Burkitt-like lymphoma with 11q aberration and $M Y C$-positive Burkitt lymphoma in the expression of CD16/CD56/CD38/CD45/CD8/CD43 by flow cytometry and CD43/LMO2/CD56 by immunohistochemistry, that may contribute to the differential diagnosis between Burkitt-like lymphoma with 11q aberration and $M Y C$-positive Burkitt lymphoma, and may help to reveal the pathogenetic mechanisms differentiating these entities. Flow-cytometry immunophenotype of Burkitt-like lymphoma with $11 \mathrm{q}$ aberration also presents similarities to that of $M Y C$ positive Burkitt lymphoma, as is mRNA and miRNA expression profiles. Given the $M Y C$-related genetic differences between these Burkitt lymphoma subtypes, probably correlated to the observed subtle but essential phenotype diversity, the similarities observed on various expression levels might suggest their common cell-of-origin following different mutational pathways. The question of mutational pathways in Burkitt-like lymphoma with 11q aberration will be the subject of our another paper (Wagener et al, in preparation). However, cases of lymphomas with $11 \mathrm{q}$ aberrations that simultaneously presented $M Y C$ rearrangements suggest a possible link between mutational pathways in Burkitt-like lymphoma with $11 \mathrm{q}$ aberration and Burkitt lymphoma.

Finally, to identify cases with a high probability of $11 \mathrm{q}$ aberration and $M Y C$ translocation, we suggest strategies based on an initial CD16/CD56 and CD38 flow cytometry immunophenotypic analysis and LMO2/CD56 immunohistochemical reaction, which may be very useful in the routine diagnostics of CD10(+) aggressive B-cell lymphomas.

\section{Acknowledgments}

We thank Elzbieta Kulczycka, MSc, for performing the immunohistochemical staining, Dr Agnieszka Giza for clinical data of patient 8, Dr Witold Gerke and Dr Tomasz Wocial for participating in the ultrasound-guided fine needle aspiration biopsy, especially in abdominal tumors and Dr Marcin Ligaj for the critical reading of the manuscript. We also thank Guillem Clot (IDIBAPS) for his help in the statistical analysis. IS was supported by the Fondo de Investigaciones Sanitarias, Instituto de Salud Carlos III (ISCIII) (Miguel Servet contract CP13/00159 and PI15/00580), the European Regional Development Fund 'Una manera de fer Europa' and 'CERCA Programme/Generalitat de Catalunya'. This work was partially developed at the Centro Esther Koplowitz (CEK), Barcelona, Spain. RS is being supported for research on pediatric lymphomas by the Kinder Krebs Initiative Buchholz/HolmSeppensen.

\section{Author contributions}

GR was the principal investigator, diagnosed cases by histopathology and immunohistochemistry, and flow cytometry, performed fine needle aspiration biopsy, drafted the manuscript, reviewed literature; BG, KB, MZ, ZB, RW, PS, and JR did the laboratory work for this study; ZB, FN, and DMG helped with presentation of the results; GR, IS, and RS evaluated and interpreted the results; KD-C, JR-J, and JW treated the pts; GR, MCh, MP-S BP-G, JW, JKS, IS, and RS, contributed to finalizing the manuscript. MCh and GR were heavily involved in writing and editing of the manuscript.

\section{Disclosure/conflict of interest}

The authors declare no conflict of interest.

\section{References}

1 Pienkowska-Grela B, Rymkiewicz G, Grygalewicz B, et al. Partial trisomy 11, dup(11)(q23q13), as a defect characterizing lymphomas with Burkitt pathomorphology without MYC gene rearrangement. Med Oncol 2011;28:1589-1595.

2 Salaverria I, Martin-Guerrero I, Wagener R, et al. A recurrent $11 \mathrm{q}$ aberration pattern characterizes a subset of MYC-negative high-grade B-cell lymphomas resembling Burkitt lymphoma. Blood 2014;123:1187-1198.

3 Ferreiro JF, Morscio J, Dierickx D, et al. Post-transplant molecularly defined Burkitt lymphomas are frequently MYC-negative and characterized by the 11q-gain/loss pattern. Haematologica 2015;100:e275-e279. 
4 Zajdel M, Rymkiewicz G, Chechlinska M, et al. miR expression in MYC-negative DLBCL/BL with partial trisomy 11 is similar to classical Burkitt lymphoma and different from diffuse large B-cell lymphoma. Tumour Biol 2015;36:5377-5388.

5 Swerdlow SH, Campo E, Pileri SA, et al. The 2016 revision of the World Health Organization classification of lymphoid neoplasms. Blood 2016;127:2375-2390.

6 Leoncini L, Raphaël M, Stein H et al. Burkitt lymphoma. In: Swerdlow SH, Campo E (eds).WHO Classification of Tumours of Haematopoietic and Lymphoid Tissues, 4th edn. IARC: Lyon, France. 2008, pp 262-264.

7 Dave SS, Fu K, Wright GW, et al. Molecular diagnosis of Burkitt's lymphoma. N Engl J Med 2006;354: 2431-2442.

8 Hummel M, Bentink S, Berger $\mathrm{H}$, et al. A biologic definition of Burkitt's lymphoma from transcriptional and genomic profiling. N Engl J Med 2006;354: 2419-2430.

9 Colomo L, Vazquez I, Papaleo N, et al. LMO2-negative expression predicts the presence of MYC translocations in aggressive B-cell lymphomas. Am J Surg Pathol 2017;41:877-886.

10 Mead GM, Barrans SL, Qian W, et al. A prospective clinicopathologic study of dose-modified CODOX-M/ IVAC in patients with sporadic Burkitt lymphoma defined using cytogenetic and immunophenotypic criteria (MRC/NCRI LY10 trial). Blood 2008;112: 2248-2260.

11 Hoelzer D, Walewski J, Dohner $\mathrm{H}$, et al. Improved outcome of adult Burkitt lymphoma/leukemia with rituximab and chemotherapy: report of a large prospective multicenter trial. Blood 2014;124:3870-3879.

12 Woroniecka R, Rymkiewicz G, Grygalewicz B, et al. Cytogenetic and flow cytometry evaluation of Richter syndrome reveals MYC, CDKN2A, IGH alterations with loss of CD52, CD62L and increase of CD71 antigen expression as the most frequent recurrent abnormalities. Am J Clin Pathol 2015;143:25-35.

13 ISCN 2016. An International System for Human Cytogenomic Nomenclature (2016). Karger: Basel: Switzerland, 2016.

14 Grygalewicz B, Woroniecka R, Rygier J, et al. Monoallelic and biallelic deletions of $13 q 14$ in a group of CLL/SLL patients investigated by CGH Haematological Cancer and SNP array (8x60K). Mol Cytogenet 2016;9: 1.

15 Maleki A, Seegmiller AC, Uddin N, et al. Bright CD38 expression is an indicator of MYC rearrangement. Leuk Lymphoma 2009;50:1054-1057.

16 Seegmiller AC, Garcia R, Huang R, et al. Simple karyotype and bcl-6 expression predict a diagnosis of
Burkitt lymphoma and better survival in IG-MYC rearranged high-grade B-cell lymphomas. Mod Pathol 2010;23:909-920.

17 Rodig SJ, Vergilio JA, Shahsafaei A, et al. Characteristic expression patterns of TCL1, CD38, and CD44 identify aggressive lymphomas harboring a MYC translocation. Am J Surg Pathol 2008;32:113-122.

18 Rymkiewicz G, Chechlinska M, Grygalewicz B, et al. Significance of a critical set of $11 \mathrm{q}$ chromosome aberrations for diagnosis of MYC negative Burkitt lymphoma. Blood 2015;126:2679.

19 Drillenburg P, Pals ST. Cell adhesion receptors in lymphoma dissemination. Blood 2000;95:1900-1910.

20 Walewski J, Domanska-Czyz K, Rymkiewicz G. Burkitt lymphoma and leukemia. Patients without HIV infection. In: Recommendations of the European Working Group for Adult ALL. In: Gökbuget N, Bassan R (eds). Recommendations of the European Working Group for Adult ALL. 1st edn. UNI-MED Verlag AG edn: BremenLondon-Boston. 2011, pp 29-40.

21 Sevilla DW, Gong JZ, Goodman BK, et al. Clinicopathologic findings in high-grade B-cell lymphomas with typical Burkitt morphologic features but lacking the MYC translocation. Am J Clin Pathol 2007;128: 981-991.

22 Rymkiewicz G, Blachnio K, Grygalewicz B, et al. Flow cytometry and cytogenetics of fine needle aspiration biopsy samples is a reliable method for diagnosing Burkitt lymphoma. Evaluation of 78 cases from a single-institution. Blood 2014;124:1640.

23 Cooper MA, Fehniger TA, Caligiuri MA. The biology of human natural killer-cell subsets. Trends Immunol 2001;22:633-640.

24 Weisberger J, Gorczyca W, Kinney MC. CD56-positive large B-cell lymphoma. Appl Immunohistochem Mol Morphol 2006;14:369-374.

25 Scholtysik R, Kreuz M, Klapper W, et al. Detection of genomic aberrations in molecularly defined Burkitt's lymphoma by array-based, high resolution, single nucleotide polymorphism analysis. Haematologica 2010;95:2047-2055.

$26 \mathrm{Wu}$ JM, Borowitz MJ, Weir EG. The usefulness of CD71 expression by flow cytometry for differentiating indolent from aggressive CD10+ B-cell lymphomas. Am J Clin Pathol 2006;126:39-46.

27 Rymkiewicz G, Romejko-Jarosinska J, Blachnio K, et al. DA-EPOCH-R is an effective regimen in high grade B-cell lymphoma defined by cell-of-origin, karyotype and BCL2/MYC/BCL6 status and expression. Blood 2016;128:1754.

28 Chisholm KM, Bangs CD, Bacchi CE, et al. Expression profiles of MYC protein and MYC gene rearrangement in lymphomas. Am J Surg Pathol 2015;39:294-303.

Supplementary Information accompanies the paper on Modern Pathology website (http://www.nature.com/ modpathol) 